Title:

A colored petri nets based workload evaluation model and its validation through Multi-Attribute Task Battery-II

Author names and affiliations.

Peng Wang (E-mail:c2t53y48@gmail.com), Weining Fang (corresponding author, E-mail:

wnfang@bjtu.edu.cn), Beiyuan Guo (E-mail: byguo@bjtu.edu.cn)

State Key Laboratory of Rail Traffic Control and Safety, Beijing Jiaotong University, No. 3 Shang

Yuan Cun, Haidian District, 100044 Beijing, China 


\title{
A colored petri nets based workload evaluation model and its validation through Multi-Attribute Task Battery-II
}

\begin{abstract}
This paper proposed a colored petri nets based workload evaluation model. A formal interpretation of workload was firstly introduced based on the process that reflection of petri nets components to task. A petri net based description of Multiple Resources theory was given by comprehending it from a new angle. A new application of VACP rating scales named V/A-C-P unit, and the definition of colored transitions were proposed to build a model of task process. The calculation of workload mainly has the following four steps: determine token's initial position and values; calculate the weight of directed arcs on the basis of the rules proposed; calculate workload from different transitions, and correct the influence of repetitive behaviors. Verify experiments were carried out based on Multi-Attribute Task Battery-II software. Our results show that there is a strong correlation between the model values and NASA -Task Load Index scores $(r=0.9513)$. In addition, this method can also distinguish behavior characteristics between different people.
\end{abstract}

\section{Keywords:}

Colored Petri Nets; Workload; Multi-Attribute Task Battery-II

\section{Introduction}

The evaluation of workload, which is a key parameter in the design and evaluation of human-machine interfaces, has an important effect on the comfort, satisfaction, efficiency, and safety of the workplace(Rubio et al., 2004). Previous studies have noted that level of workload is related to accident frequency(Lei et al., 2009) and job performance(Brookhuis et al., 2009). In the conference, 'Workload: Its Theory and Measurement,' in 1977, researchers taught that workload was a multi-dimensional concept: it involves work requirements, time pressure, ability of the operator, behavioral expression, and many other factors(Moray, 2013). After decades of research and development, the definition of workload has changed and is now more interpreted in terms of demanded resources on time and cognition when performing a task(Pickup et al., 2010; Shneiderman, 2012).

Methods for evaluating workload can be divided into three types: subjective self-report measures, performance measures, and physiological measures(Silva, 2014). Subjective self-report measure are implemented by questionnaires or interviews, such as the NASA-Task Load Index (NASA-TLX)(Hart and Staveland, 1988), Subjective Workload Assessment Technology(Reid and Nygren, 1988), Multiple Resources Questionnaire(Boles and Adair, 2001), and Integrated Workload Scale(Pickup et al., 2005). Performance measures employ two main types of measurement, primary task measures(Bromfield and Dillman, 2015)and secondary task measures (Pauzié and Manzano, 2007), and use time or error rate as the calculation basis to evaluate workload. Physiological measures evaluate signals of the peripheral and central nervous system of participants, and their major measurements include Heart Rate Variability(DiDomenico and Nussbaum, 2011), Electroencephalography(Zarjam et al., 2013), and Eye Movement(Brookhuis and de Waard, 2010). Nevertheless, several researchers think that workload has a multidimensional indicators, a single evaluation method cannot reflect all aspects of workload. Such researchers have proposed a comprehensive evaluation model to solve the problem, including Overall Workload Level (OWL)(Jung and Jung, 2001)and ACT-R cognitive architecture(Jo et al., 2012).OWL examines four variables: physical job demand, environmental factors, postural discomfort, and mental job demand. In one particular study utilizing OWL, 28 workers gave a mark to each variable using a five-point value, and the analytic hierarchy process (AHP) was then applied to estimate the external workload. ACT-R cognitive architecture was originally used to evaluate operators' performances, and workload was measured by an algorithm that calculated the activated time of the ACT-R modules.

Table 1

VACP rating scales.

\begin{tabular}{ll}
\hline Rate & Description \\
\hline Visual & \\
0.0 & No visual activity(V0) \\
1.0 & Visually register/detect (detect occurrence of image)(V1) \\
3.7 & Visually discriminate (detect visual differences)(V2) \\
4.0 & Visually inspect/check (discrete inspection/static condition)(V3) \\
5.0 & Visually locate/align (selective orientation)(V4) \\
5.4 & Visually track/follow (maintain orientation)(V5) \\
5.9 & Visually read (symbol)(V6) \\
7.0 & Visually scan/search/monitor(continuous/serial inspection, multiple conditions)(V7) \\
Auditory & \\
0.0 & No auditory activity(A0) \\
1.0 & Detect/register sound (detect occurrence of sound)(A1) \\
2.0 & Orient to sound (general orientation/attention)(A2) \\
4.2 & Orient to sound (selective orientation/attention)(A3) \\
4.3 & Verify auditory feedback(detect occurrence of anticipated sound)(A4) \\
4.9 & Interpret semantic content(speech)(A5)
\end{tabular}




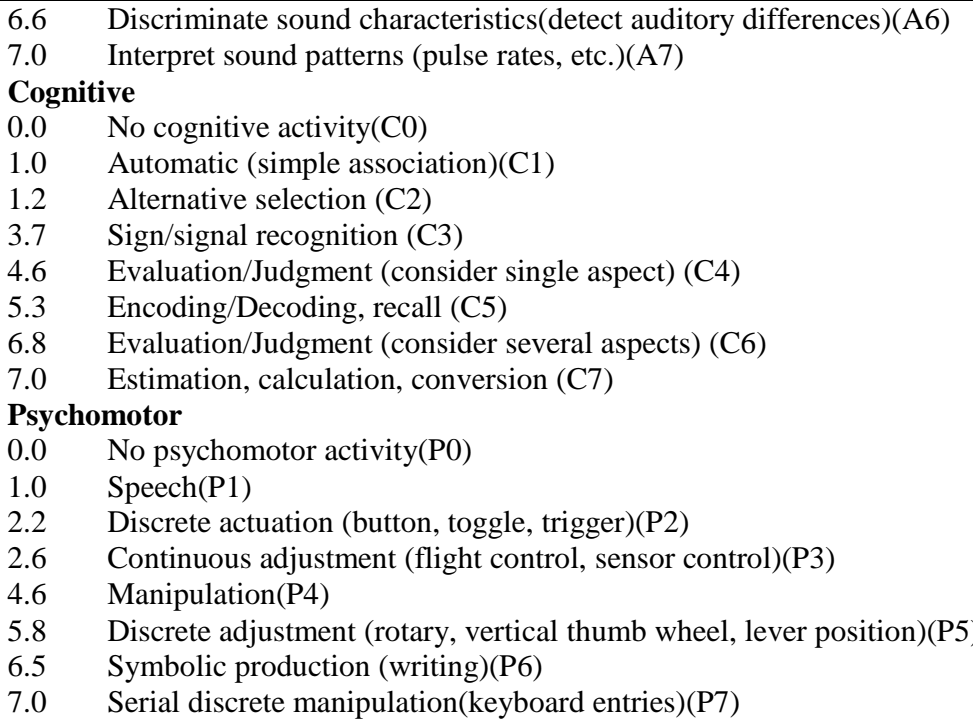

To focus exclusively on the workload of helicopter pilots, the VACP rating scales method was proposed(McCracken et al., 1984).VACP has four parts: visual demand, auditory demand, cognitive demand and psychomotor demand. Every part defines several abstract behaviors that are encoded (shown in Table 1), and the ratings range from 0 to 7 . The main advantage of the VACP rating scales method was that it measured the demand on resources from basic abstract behaviors. Therefore, workload could be predicted after decomposition of the task to the behavioral level. Some researchers(Hamilton and Clarke, 2005; Wang and Fang, 2014) have used the VACP rating scales to evaluate codes of behavior in driver's performance, modeling and error prediction. As a standard of measure(Keller, 2002),VACP rating scales were applied to predict the workload of driving a car while talking on a cell phone, and the evaluation of the workload was performed by calculating the maximum, mean, and standard deviation of the task's total demand for resources after analysis of the task. To verify the effectiveness of VACP rating scales(Liang et al., 2014), a comparison was performed between the predicted results of VACP ratings scales and a NASA-TLX questionnaire that were given in a study that measured the workload of physical therapists. The task analysis was based on the physical therapists' standard operating procedures and discussion with the therapists. The correlation between the VACP ratings scales and NASA-TLX questionnaire was higher than 0.7. Although this method has been widely used, some issues should be noted:

- First, the methods for applying the VACP rating scales were not unified. Workload calculation can be divided into two steps: Determine the behaviors of the tasks according to VACP rating scales, and then calculate them through a set of rules. Expert discussion guides the first step, while different sets of rules have been proposed for the second step. For instance, one such set of rules suggested determining the behaviors in different phases of the task, and then adding the values in four dimensions: visual, cognitive, auditory and psychomotor(Liang et al., 2014); however, this method was completely subjective and ignored the influence of time. Another set of rules considered the conflict of concurrent interface channels(North and Riley, 1989). Finally, another set of rules proposed calculating workload by time occupancy ratios(Popkin et al., 2001). Some researchers used formal tools to analyze the tasks that they do not evaluate through the behavioral aspects(Mcllroy and Stanton, 2015).

-Second, continuous repetitive and dispersive repetitive behaviors have different influences on the evaluation of workload. This finding was made obvious in certain behaviors that focused on interaction with the same object. For instance, consider an experiment we did that involved the repetitive task of pressing a button. The operator needed to press the button 3 times in the task procedure at different time intervals. The behaviors we recorded were three psychomotor P2 (shown in Table 1), and we added these resources demanded to the other behaviors to predict the workload. However, if the procedure involved pressing the button three times continuously (as opposed to at different time intervals), the resources demanded were less than three psychomotor because human will recognize the operating mode in the first process of the operation.

The solution method proposed was based on Colored Petri Nets (CPN). Colored petri nets, in other words, assign some attribute to place, transition, tokens, and thus the model can represent more things in a brief way. Although CPN has not been directly used for workload assessment for its initially application that as a kind of formal analysis tools, and the definition of the components and analysis level of petri nets in previous studies, were not clear, four reasons are proposed there for its good potential in workload assessments.

Firstly, petri nets, the base net of CPN, has been applied to a variety of analyses and modeling of ergonomic tasks(Kontogiannis et al., 2000; Mascaro and Asada, 1998; Wen and Hwang, 1999), and task analysis and modeling is the essential part especially in some workload assessments that without human-in-the-loop. In 2005, a petri nets based approach was proposed for ergonomic task analysis and modeling with emphasis on adaptation to system changes(Kontogiannis, 2005, 2003). In the resulting paper, workload was regarded as one of the causes of system change. In this study(Yagoda and Coovert, 2012), petri nets modeling was used to evaluate the workload of search-and-rescue unmanned aerial vehicle operations. Before the petri nets modeling performed, a job analysis and cognitive work analysis of the tasks were performed. From the ensuing petri nets model, a place represented a state in the process of task execution; a transition 
represented a set of operations in action; and other petri nets components, such as the weight of directed arcs and tokens, were not defined.

Next, for a more accurate assessment, a clear definition of workload should be previously given. However, no unified expression of workload has been reached in the past few decades. There are three characteristics could be found in the definition of workload in previous studies: multi-dimension, task demand and resource (ability) of human.

-Multi-dimension: multi-dimension is reflected in two aspects. The first one is that workload depends on specific human, machine, environment and task, and the assessment of work should combine them. An example proposed is the six dimensions of NASA-TLX. The mental demand, physical demand and temporal demand are the description of specific machine, environment and task, and the performance, effort and frustration are the description of specific human. Another one is that workload can be applied to human from multiple aspects. For instance, VACP ratings scales divided workload into four parts: visual, auditory, cognitive and psychomotor. The multiple resources theory(Wickens, 2002) evaluates workload in three aspects: time pressure, behavioral conflict and cognitive demand.

- Task demand: Task is the motivation of human-machine interaction that generates workload, and task types often leads to different workload definition. Some descriptions are: total attention demand(de Winter et al., 2014; Di Stasi et al., 2011); specific task demand(DiDomenico and Nussbaum, 2005).; the specific attention demand needed to reach a certain performance of task(Benedetto et al., 2011; Overmeyer et al., 2016).

- Resource (ability) of human: For the reason that human is the objective carrier of workload, the resource (ability) of human has been often mentioned in the definition of workload. In the research that information processing between man and driving assistance system, Brookhuis et al(Brookhuis et al., 2009)defined mental workload as the specific ability in task execution. Liang et al(Liang et al., 2014)regarded workload as the demand resource in information processing of a task.

Consequently, for a more accurate assessment of workload, we suggest that the definition of workload should be transformed from a literal expression to a symbolic expression, and finally to a formulate expression. Exactly, CPN is a suitable method of symbolic expressing workload.

In addition, as an assistant tool, task analysis methods have influence on the accuracy of workload assessments. The different elements in task analysis methods will lead to different results. The elements in hierarchical task analysis(Al-Hakim et al., 2014) are "goal", "task", "plan" and "operation", while the GOMS (Goals, Operators, Methods, and Selectors)(Oyewole and Haight, 2011)consists of "goal", "operator", "method" and "selection rule". CPN has the ability that integrating related concepts and elements needed for workload description in one structure, and has a better unity than the other task analysis methods.

Lastly, some other characteristics also benefit the assessments of workload. The concrete definition and parameters of petri nets components can be changed when needed; therefore, VACP rating scales can be combined with petri nets for modeling workload. Petri nets have the ability to describe concurrent systems; thus, it can be used to describe the two-channel cognitive process of multiple resources theory. Petri nets can describe different interactive behaviors with a variety of structures, including 'and,' 'or,' 'in parallel,' or 'serial'.

In this paper, the specific method is proposed in the second section, and the verification experiments, based on Multi-Attribute Task Battery-II (MATB-II), is put forward in the third section.

\section{Material and methods}

\subsection{Modeling of task process}

\subsubsection{Mapping petri nets components to task}

For the purpose of evaluating workload based on colored petri nets, the first step is mapping petri nets components to tasks. In this paper, a transition represents a type of interaction behavior, and the behavior aggregate is shown in Table 1. A place represents a type of interaction with objects, and the standards for determining place were as follows:

-Place is an abstraction, a type of specific interaction with objects.

- The behavioral interaction between human and place is direct.

-Distinctions between different abstractions in the specific interaction with objects are made if there are different interactive behaviors.

Directed arcs represent the direction of the process of a task. Tokens correspond to the resources of human. Consequently, the explanation of workload by petri nets can be described as the consumption of resources (tokens) being driven by interaction behaviors (transitions) that are the result of a subject's interaction with objects (places). 


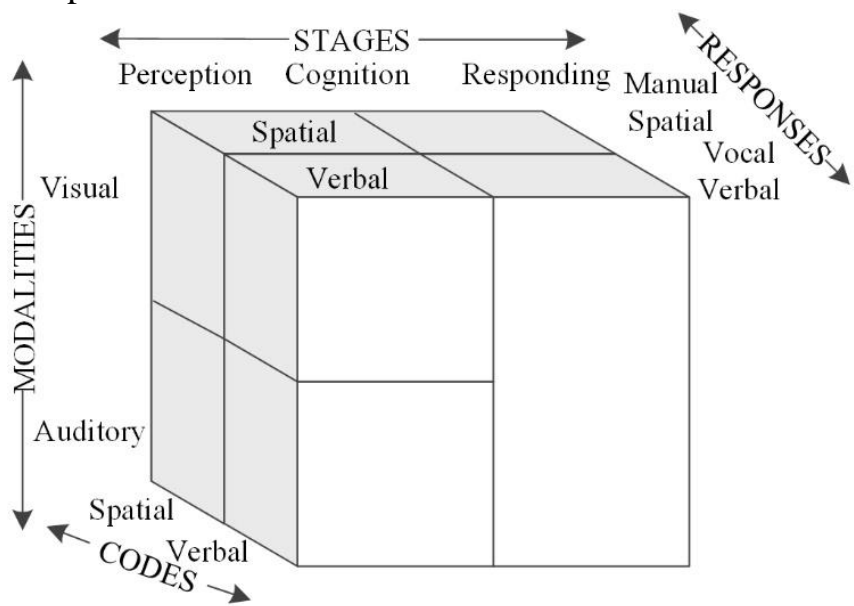

Fig. 1. Representation of the structure of multiple resources

According to the representation of the structure of multiple resources (based upon multiple resources theory) in Fig. 1(Wickens, 2002), interaction processes can be divided into three stages: perception, cognition and response. Therefore, combined with the VACP rating scales, a complete simple interaction process can be considered as a complete V/A-C-P unit, and the determination of the number of interaction behaviors and calculation of workload should be based on this.

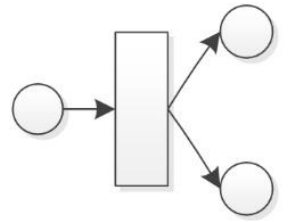

AND-split(fork)

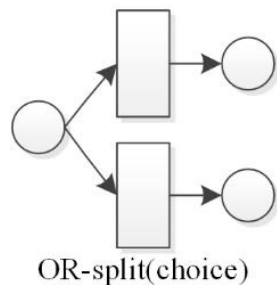

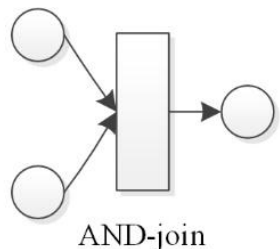

AND-join (synchronization)

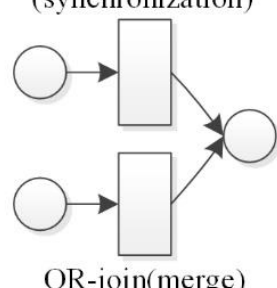

Fig. 2. Four types of Petri nets structure

Another point that can be taken from Fig. 1 is that each of the three stages of the interaction process has two channels, that is, human-machine interaction can be a parallel process. Petri nets have the ability to describe these parallel processes. As shown in Fig. 2, these four types of petri net structure can be interpreted as the abstraction of specific interaction behaviors. For instance, the AND-split (fork) and the AND-join (synchronization) represent the beginning and end of a parallel interaction process. In the OR-split (choice) and the OR-join (merge) structure, tokens can exist in multiple places. Consequently, these two structures can also be interpreted as a parallel interaction process.

A petri net based representation of the structure of multiple resources from the view of information processing is shown in Fig. 3. The input information can be divided into visual components and auditory components. After the perception, the state of information turns into 'To be encoded,' and this process is in a AND-split (fork) structure; this is because after visual access the state of a visual/auditory component of information can also become 'To be spatially encoded' or 'To be verbally encoded.' After it is encoded, the state of information turns into 'To be responded to', and here also it uses a AND-split (fork) structure to describe the process. Eventually, after spatial/verbal responses, the output information that consists of spatial and verbal components could be determined. Although not all input information consists of both visual and auditory components and not all output information consists of both spatial and verbal components, this structure was still available when the corresponding place and transition were removed.

An example of a common interaction process is given in Fig. 4. When an operator observed a warning icon, he pressed a button as a response, and he needed to press the button repeatedly when the warning icon appeared again. From the perspective resource flows, tokens will move from the place (interaction with an object) 'warning icon' to the place 'press button' repetitively. However, this conflict with petri nets theory because the amount of tokens in the place 'warning icon' is limited, and from the point of specific interaction phenomena, operators' eyes are still on the warning icon when they are familiar with the operations. 
Fig. 3. Petri nets based representation of the structure of multiple resources from the view of information processing

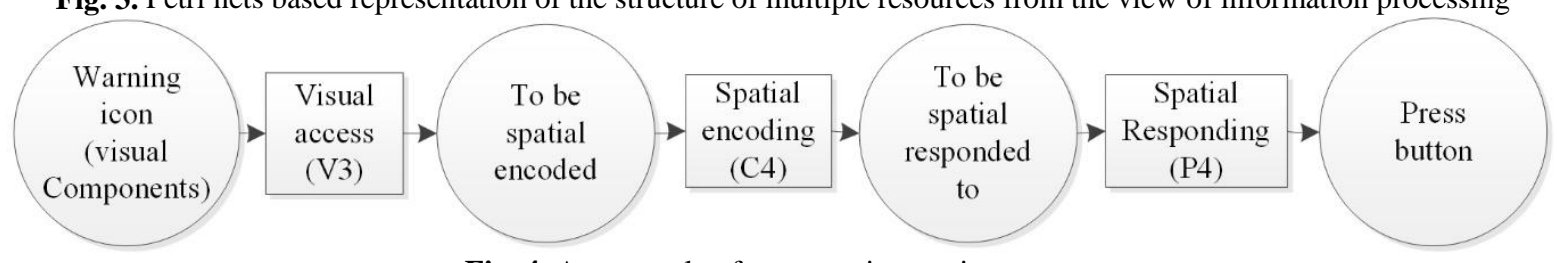

Fig. 4. An example of common interaction process

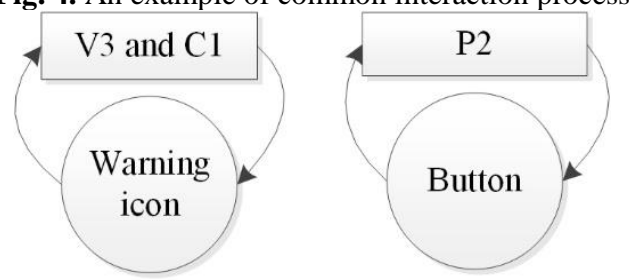

Fig. 5. Warning feedback interaction process from the perspective of resource flows

As shown in Fig. 5, a model of warning feedback interaction processes (from the perspective of resource flows) was given to adapt petri nets theory. A self-loop structure with a place and a transition was set up to give an explanation to resource flows. A transition comprises of two sub-transitions, visual access (V3) and spatial encoding (C1), and two sub-places were connected with the place 'warning icon' by a directed arc. Thus, instead of constantly consuming resources, tokens would be in a consumption-regeneration process. The self-loop structure was repeated for the task of manipulating a button in the same way. In a process in which there is constant interaction with an object, workload can be interpreted as a result of this consumption-regeneration process with tokens, while for a multi-object interaction process, workload can be interpreted as a result of the transfer of tokens.

\subsubsection{Structure and amount of V/A-C-P units}

When completing a mapping task for petri net components and representing task processes on petri nets based upon consumption-regeneration or transfer processes, a petri net structure of tasks can be established by the following two steps.

The first step is to record psychomotor and visual/auditory interactions, and also the values of the interaction with the object. Psychomotor activities, such as clicking a mouse or pressing button, can be recorded by a computer, while other psychomotor activities can be recorded by video recorder. Visual interactions can be recorded by an eye tracker, and auditory interactions can be recorded by a digital recorder. The types of interactions in Table 1 were determined through expert discussion.

The second step is to build the petri nets structure of tasks based on V/A-C-P units in section 2.1.2. This task analysis process consists of two parts that determine the form of expression of the V/A-C-P units in the petri nets and that ascertain the amount of each transition. Seven types of V/A-C-P units are given in Fig. 6 to express different interactions. Their descriptions are as follows:

Fig. 6(a): A single process in which all interactions are with the same object A.

Fig. 6(b): Information obtained from perceiving and cognizing on object A while executing a process on object B simultaneously, such as the process of maintaining the speed limit while driving.

Fig. 6(c): Information obtained from perceiving and cognizing on object A after P process has been executed on object B lately.

Fig. 6(d): Like Fig. 6(c), the difference is that it is executed on objects B and C simultaneously. This represents a type of parallel structure due to the limited space of this paper, and execution of tasks on the object may actually be more than B and $\mathrm{C}$. The following three types were the same.

Fig. 6(e): Information obtained from perceiving and cognizing on object A while executing a process on object B and on object $\mathrm{C}$, sequentially.

Fig. 6(f): Like Fig. 6(c), the difference is that information is obtained from object A and B, respectively, and it is performed through a comprehensive cognitive process (or a comprehensive cognitive process with a $\mathrm{P}$ process). 
Fig. 6(g): Information obtained from perceiving and cognizing on object $\mathrm{A}$ while executing a process on object $\mathrm{C}$ in one instance, and then information obtained from perceiving and cognizing on object B while executing a process on object 1

2

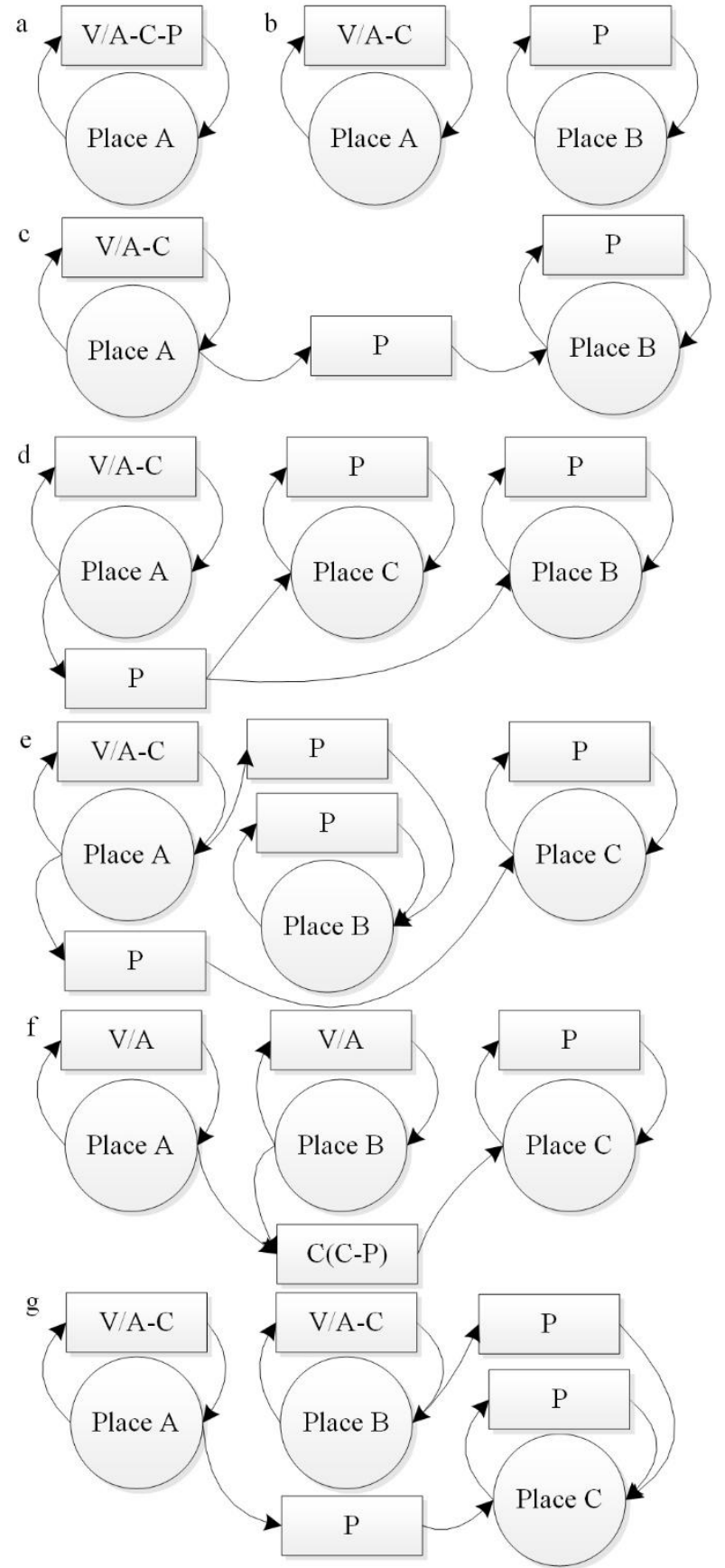

Fig. 6. Seven types of V/A-C-P units

The amount of each transition can be calculated from the amount of psychomotor activity. This finding is observed because psychomotor activities have relatively more accurate records than other behaviors, and every psychomotor that contributes to workload is in a V/A-C-P unit. Because certain psychomotor activities consist of a series of actions, such as consecutive mouse clicks, they should be observed as one. The amount of transitions is determined by the amount of $\mathrm{P}$ that it contains. When a transition does not contain a $\mathrm{P}$, such as V/A, V/A-C or $\mathrm{C}$ for which the rules stipulate that every psychomotor activity has a V/A-C-P unit, their amount is equal to the corresponding transition that has a P. Finally, the amount of psychomotor activity can be calculated based on the values established from the interaction with objects. The method was given as follows:

-If the value of an object is a switching value: the psychomotor activity is recorded one time when the value of object changes.

-If the value of an object is numerical: according to the method here (Chen et al., 2013), given a constant $N$, sequential data inputs for an object are $X=\left\{x_{1}, x_{2}, \ldots x_{n}\right\}$, the mean $\bar{m}$, and the standard deviation scan be calculated as Eq. (1) and Eq. (2): 


$$
\begin{gathered}
\bar{m}=\frac{\sum_{i=1}^{N} x_{i}}{N} \\
s=\sqrt{\frac{1}{N-1} \sum_{i-1}^{N}\left(x_{i}-m\right)^{2}}
\end{gathered}
$$

Let $D_{\max }$ represent the maximum difference between the values of an object in $N$ sequential data, calculate the number of ranges that for $x_{\mathrm{i}}$ are within these ranges, the value of $x_{\mathrm{i}}$ is out of $\left(\bar{m}-\max \left(s, D_{\max } / 10\right), \bar{m}+\max \left(s, D_{\max } / 10\right)\right)$. The number of ranges was considered to be the amount of psychomotor.

Two special cases should be illustrated here as a supplement:

- The tasks process seemingly had no psychomotor: An example extended from the mentioned warning feedback interaction process that was given here: the operator pressed the button when he saw the right warning icon, and when he saw a wrong warning icon, he did not need to press button. It seems the behavior in this process is just V-C. The amounts of transition V-C can be calculated by recording the number of times the other warning icons appeared.

Another example can be taken from a task that had subjects try to remember three numbers that appeared on a screen. This task seemingly just had visual behavior, but it was not a complete interactive process. It lacked a process that would validate the result of memory. Workload can be calculated when the interaction process of the task is completed, and the process that validates the result of memory has some psychomotor activity, which can be calculated by the method given above.

- Psychomotor in the tasks was just Speech (P1): For example, subjects were asked to talk about the members of their family and also asked to discuss their understanding of ergonomics. Both of the tasks had the same required time, three minutes. Although they have the same psychomotor, workload values still can be distinguished by their different cognitive behaviors; that is, automatic (simple association) (C1) and estimation, calculation, and conversion (C7).

\subsubsection{Colored transitions}

Colored transitions have the same effect on Colored tokens which in traditional colored petri net that can make complex petri nets become simple and contain more complex meaning. They were used to describe the interaction behaviors in this workload model. The four parameters of transitions are as follows:

- Change in types of resource: Resources was divided into two types, resources transfer (RT) and resources recycle (RR). When RR happens, resources were circling in the same place, and when RT happens, resources were transferring from one place to the others.

- Number of sub-Transitions: As shown in Fig. 4 and Fig. 5, the number of sub-transitions was determined by specific interactions and rules for tokens in petri nets.

-Behavior type: From the VACP rating scales in Table 1.

- Execution sequence: Indicate the execution sequence in the behavior of sub-transitions in the interaction process.

It was noted that the values in Table 1 were multiplied by ten for ease of workload calculation. An example of a colored transition is shown in Fig. 7, and the change type of resource for this transition was resources transfer. It has three sub-transitions, and the execution sequence was 40 . The first one's behavior type was $\mathrm{C} 1$; the second one's behavior type was P2; and the third one's behavior type was P1.

$$
\text { RT;3(C1-P2-P1:40) }
$$

Fig. 7. An example of colored transition

\subsection{Workload calculation}

\subsubsection{Initial position and value of tokens}

For the detailed description of the workload through the CPN model, the initial position and value of tokens were given here. The initial position of tokens was determined by the initial interaction with the object at the beginning of the task, and the initial value of tokens was equal to the demand resource of the first interaction behavior. An example of the same warning feedback interaction process was shown in Fig. 8. When this task began, the first interactive behavior with the button was P2; hence, the initial position of tokens was in the place 'button'. The initial value of tokens was 22, for the demand resource of P2 was 22 .

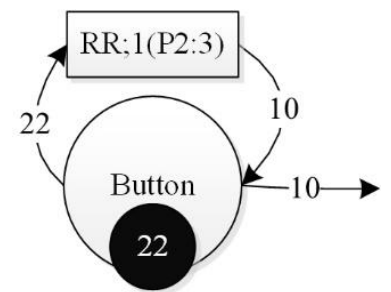

Fig. 8. An example of the initial position and value of tokens 


\subsubsection{Rules and calculation of directed arc's weight}

For the allocation of tokens to different places at the trigger moment of a transition, the directed arc's weight was set up

1

2

to make the CPN model accord with the actual interactions. The six rules for determining the directed arc's weight are given here:

-Directed Arc's weight was related to the execution process of a task and different interaction behaviors. Thus, it was sequentially determined from a token's initial place according to the execution process of the task.

- The basis of generation for interactive behaviors was that the demanded resource was sufficient. In the CPN model, it appears that the sum of the transition's input and directed arc's weight was equal to the demand resource of the first sub-transition in it.

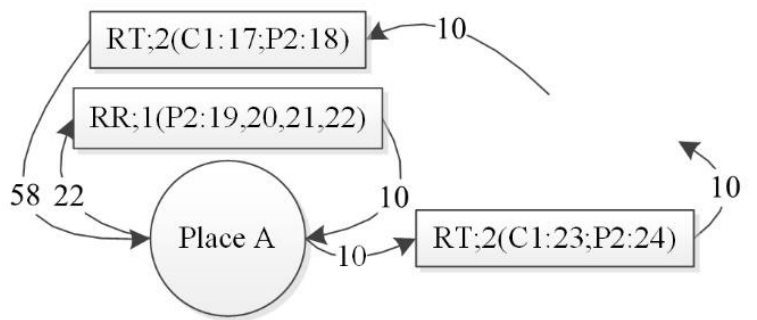

Fig. 9. A case of CPN model that resources completely transfer from place A

-Directed arc's weight was related to whether resources completely transfer from one place to the others. In some cases, such as Fig. 9, for the operator, it was no longer the other interactions after P2 with Place A; thus, resources will completely transfer from it when RT;2(C1:17;P2:18) is executed. While for the interaction in Fig. 10, resources transferred incompletely from Place A, for the part of resources in Place A were in a 'remaining state.' This 'remaining state' means that the operator was focused on the object without any interactions. In Fig. 10, after RT;1(P2:15) was executed, the operator began to interact with Place B, and 10 tokens were in the 'remaining state' before RT;2(C1:17;P2:18) was executed.

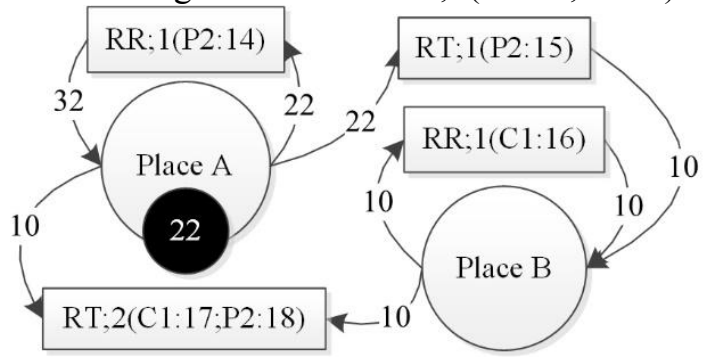

Fig. 10. A case of CPN model that resources partly transfer from place A

- For the description of repetitive behaviors, two specific rules for determining the place's input directed arc's weights are given as follows:

(1) A self-loop structure with a place and a transition that has been executed several times: As shown in Fig. 9, the transition RR;1(P2:19,20,21,22) has been executed for 4 times, and its input directed arc's weight was 22 for the first interaction behavior P2. Place A's output directed arc's weight was 10 for the first interaction behavior $\mathrm{C} 1$ in the transition $\mathrm{RT} ; 2(\mathrm{C} 1: 23 ; \mathrm{P} 2: 24)$. For this transition, after the interaction with A, resources completely transfer from it. The input directed arc's weight of Place A was 10. While, resources reduced to 12 when the transition RR;1(P2:19,20,21,22) was executed one time; thus, the demanded resources for the execution of RR;1(P2:19,20,21,22) was for four times, and added with the demanded resources for the execution of RT;2(C1:23;P2:24), the directed arc's weight between transition RT;2(C1:17;P2:18) and Place A will be calculated as $12 \times 4+10=58$.

(2) A self-loop structure with a place and a transition that has several groups of repetitive executions: As shown in Fig. 11, if the transition RR;1(P2:19,20,21,22) in Fig. 9 changes to RR;1(P2:19,20,21,22,56,57), 58 is not equal to the demand resource for it; thus, an additional transition and directed arc should be added to meet the demand resource. The additional value was $12 \times 2+10=34$.

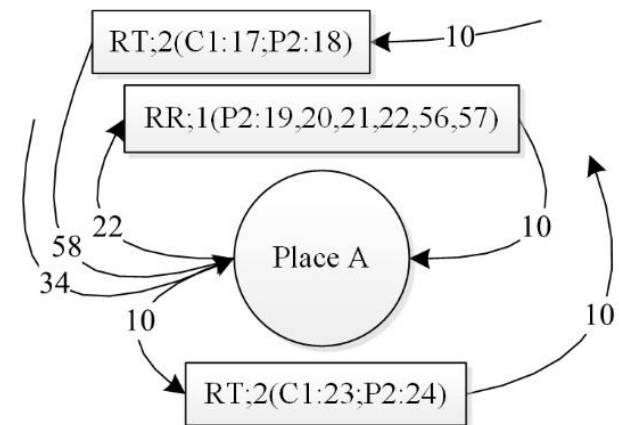

Fig. 11. A case of CPN model that a self-loop structure with a place and a transition has several groups of repetitive execution

- The method for determining a directed arc's weight between sub-transitions and sub-places was similar to how we determine transitions and places for their sequentially connected structure. 


\subsubsection{Calculation about transitions and directed arc's weight}

As mentioned in 2.2.1, interaction behaviors led to workload, and they were described as transitions in the CPN model.

Thus, the parameters of colored transition and directed arc's weight constitute the calculation parameters of workload. The computing method was given as Eq. (3), $A_{\mathrm{ij}}$ was the $j$ th weight belonging to $m$ output directed arcs of the transition; $A_{\mathrm{ik}}$ was the $k$ th weight belonging to $n$ input directed arcs of the transition; $T_{\text {ip }}$ was the demand resource of $p$ th belonging to $q$ sub-transitions of the transition.

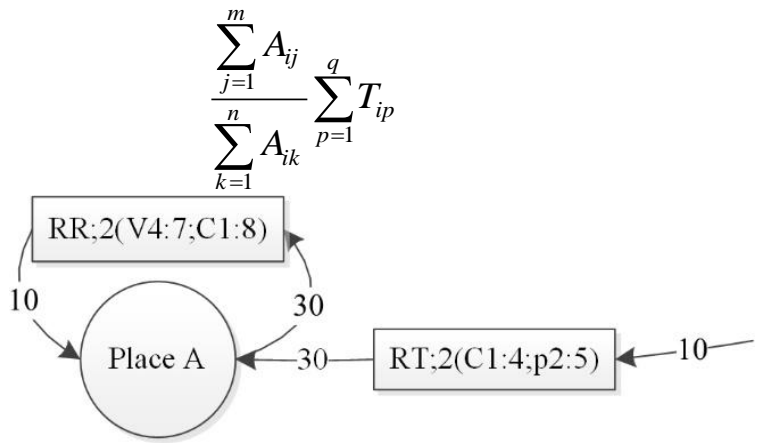

Fig. 12. Two types of transitions

There were two types of transitions from the perspective of the CPN structure form, self-loop and normal. For instance, see transitions RR;2(V4:7;C1:8) and RT;2(C1:4;P2:5) in Fig. 12. This paper supposes that these two structures have different influences on workload calculation. For a task that has self-loop transitions executed $a$ times and normal transitions executed $b$ times, their influence coefficient for workload were $a / a+b$ and $b / b+a$ respectively.

2.2.4. Influence of repetitive behaviors to workload

In task execution process, such as the warning feedback task in Fig. 4, some interaction behaviors needed to be repeated many times. This paper suggests that continuous/dispersive repetitive interaction behavior will cause different influences on workload. Assume that a transition was executed for $k$ times, and the order of the execution was $\left\{n_{1}, n_{2, \ldots} n_{\mathrm{k}}\right\}$. For $n_{\mathrm{j}}$ in the $k-1$ executed transitions, except $n_{1}$, the influence coefficient of workload because of repeat behaviors was $n_{\mathrm{j}}-n_{\mathrm{j}-1} / n_{\mathrm{j}}-n_{1}$.

2.2.5. Calculation formula

$$
\frac{\frac{a}{a+b} \sum_{i=1}^{a}\left(\frac{\alpha_{e}-\alpha_{e-1}}{\alpha_{e}-\alpha_{1}} \frac{\sum_{j=1}^{m_{i}} A_{i j}}{\sum_{k=1}^{n_{i}} A_{i k}} \sum_{p=1}^{q_{i}} T_{i p}\right)+\frac{b}{a+b} \sum_{r=1}^{b}\left(\frac{\beta_{f}-\beta_{f-1}}{\beta_{f}-\beta_{1}} \frac{\sum_{s=1}^{t_{r}} A_{r s}}{\sum_{u=1}^{v_{r}} A_{r u}} \sum_{w=1}^{z_{r}} T_{r w}\right)}{T_{\text {(min) }}}
$$

Eq. (4) was the computing method of workload based on the CPN model; the parameters were defined as follows: $a$ : Total number of execution times of self-loop transitions.

$b$ : Total number of execution times of normal transitions. $m_{\mathrm{i}}$ : Number of the output directed arc of $i$ th self-loop transition.

$n_{\mathrm{i}}$ : Number of the input directed arc of $i$ th self-loop transition.

$q_{\mathrm{i}}$ : Total demand resource of sub-transitions belong to $i$ th self-loop transition.

$t_{\mathrm{r}}$ : Number of output directed arcs of $r$ th normal transition.

$v_{\mathrm{r}}$ : Number of input directed arcs of $r$ th normal transition.

$z_{\mathrm{r}}$ : Total demand resource of sub-transitions belong to $r$ th normal transition.

$p$ : $p$ th sub-transition belong to $i$ th self-loop transition.

$w$ : $w$ th sub-transition belong to $r$ th normal transition.

$\alpha_{e}: e$ th of $c_{\mathrm{i}}$ repeated times of $i$ th self-loop transitions.

$\beta_{f}: f$ th of $d_{\mathrm{r}}$ repeated times of $r$ th normal transitions.

$T_{(\min )}$ : The execution time of the task, the unit is minutes.

\section{Experiments}

\subsection{Participants}

Twenty-eight students participated in the study (18 males and 10 females, 13 undergraduates and 15 postgraduates). Their ages ranged from 20 to $25(M=22.75, S D=1.50)$. Participants had no prior contact with the software and hardware used in the experiments, or had known about the background. They were asked to rule themselves out if they had a physical disease or mental abnormalities, and they signed an agreement to ensure banning on alcohol or coffee on the night before the experiments. 
Experiments were implemented between nine and eleven in the morning or three and five in the afternoon in a quiet 1 indoors room with ambient air, soft light and a $25^{\circ} \mathrm{C}$ comfort temperature. A CORE-i7 computer with a 22-inch LCD monitor 2 and a standard keyboard, as well as headphones, a joystick, a mouse, and an SMI iView X RED eye tracking system were used 3 in the experiments.

The test environment used for these experiments was the Multi-Attribute Task Battery-II(Santiago-Espada et al., 2011).It was the next generation of Multi-Attribute Task Battery(MATB)(Comstock and Arnegard, 1992).This multitasking environment had been used in several previous studies as a tool for evaluating workload(Gonzalez, 2005; Wang et al., 2012).As shown in Fig. 13, MATB-II consisted of four sub-tasks, which consisted of different combinations of sub-tasks that can be set by changing the parameters, like time and the number of events. A NASA-TLX questionnaire was embedded in this software for assessment of the subjective workload. The explanation of the sub-tasks was as follows:

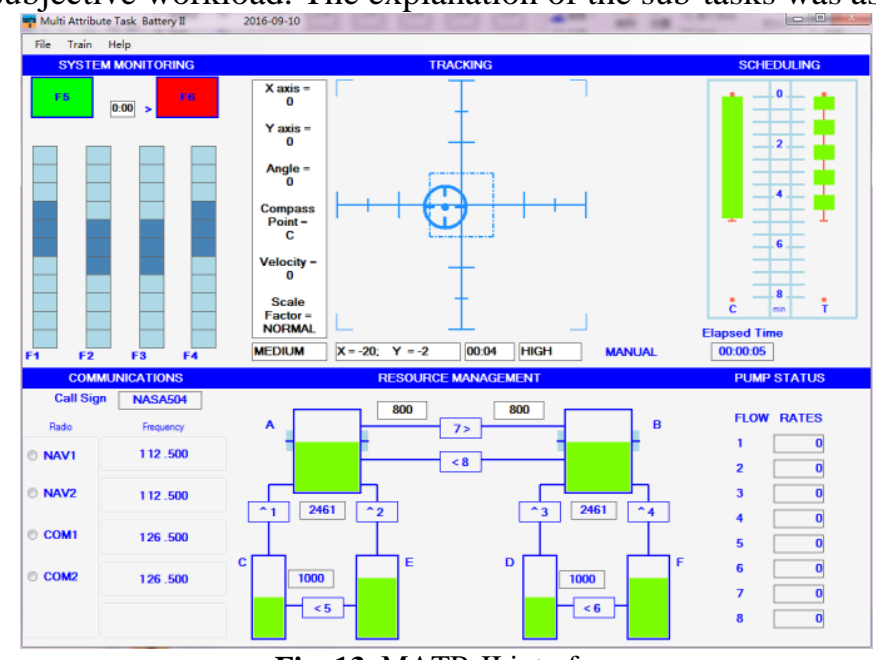

Fig. 13. MATB-II interface

System Monitoring (SYSMON): Participants pressed the corresponding key, or clicked the icon when a green F5 icon turned off or red F6 icon turned on, or the indicator F1/F2/F3/F4 moved up and down independently on each value.

Tracking (TRK): In MANUAL model, participants kept the target in the center of the inner box through a joystick. In AUTOMATION model, participants did not need to do anything.

Communication (COMMUN): Participants adjusted the corresponding frequency of the channel according to the voice when he listened to 'NASA 504'.

Resource management (RESMAN): Participants adjusted eight pumps to keep the level of fuel in Tank A and Tank B within 200 ranges of 2500 , and pumps could not be operated when they were in a fail state.

\subsection{Tasks}

Each task had the same execution time of $5 \mathrm{~min}$. There were three levels of difficulty for these four sub-tasks; they were put forward in Table 2 according to a preliminary experiment, and the calculation model was proposed in section two. For SYSMON tasks, the difference of difficulty was mainly manifested on different number of warnings. Difficulty was distinguished by the proportion of MANUAL states in $5 \mathrm{~min}$ for TRK tasks. Difficulty of COMMUN tasks was distinguished by changing the amount of speech. For RESMAN tasks, difficulty was determined by changing the number of pumps that fail and fix simultaneously. Combining every two, every three sub-tasks, and twelve single tasks in three levels, forty-two different tasks were set up to build a task library for the experiments. It was noted that the warnings in the SYSMON tasks, the voice in the COMMUN tasks, and the fail pumps in the RESMAN tasks were randomly selected in case participants found any rules.

Fourteen tasks were selected from the task library for final experiments; they were COM5, RES2, TRK60\%, SYS45, RES2\&TRK60\%， COM5\&RES1， COM15\&SYS30， SYS15\&TRK20\%， COM25\&TRK100\%， RES3\&SYS45, SYS15\&RES1\&TRK20\%, RES2\&TRK60\%\&SYS30, RES2\&TRK60\%\&COM15, COM25\&RES3\&TRK100\%. Four of them were a single sub-task, six were double sub-tasks combinations, and another four were treble sub-tasks combinations. These tasks also included different difficulty levels of sub-tasks and different types of sub-tasks: four of them were level1; six were level2; and another four were level3. SYSMON tasks had appeared six times; TRK tasks had appeared eight times; COMMUN tasks had appeared six times; and RESMAN task had appeared eight times.

Five different tasks were randomly selected from the fourteen tasks for each participant. This process was through a program in which each of the fourteen tasks was executed ten times.

Table 2

Three levels of difficulty of these four subtasks.

\begin{tabular}{ccccc} 
Difficulty & SYSMON & TRK & COMMUN & RESMAN \\
\hline Level1 & SYS15:15 warnings & TRK20\%:5 groups consist of a 12s & COM5:3 & RES1:1pump in pump 1-8fail and fix
\end{tabular}




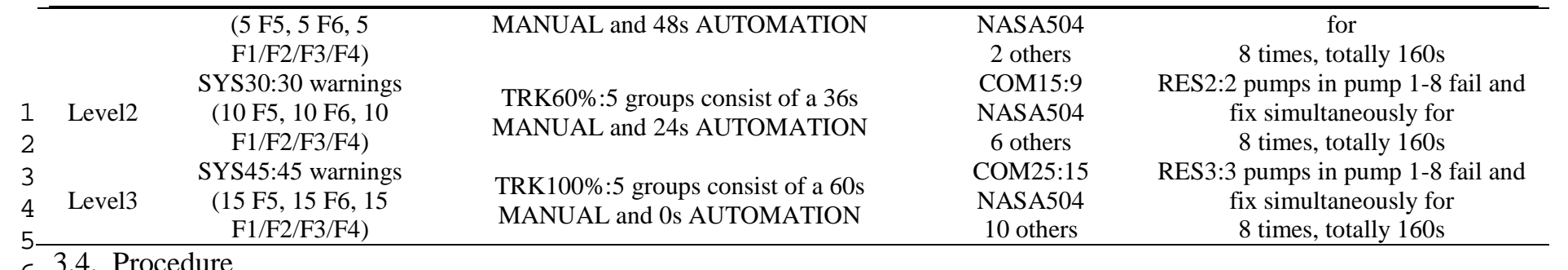

Each participant took approximately 75 minutes to complete their experiments. First, the participants signed an informed consent, and those whom were allocated to execute task COM5 were additionally asked to record their College English Test (CET) levels. Next, they were briefed about the nature of the experiments, and completed a 10 min training section, including the two tasks TRK20\% and COM25SYS45RES3. These two tasks were the simplest and the hardest one in the task library, respectively, from the preliminary experiments. Another purpose of the training session was that it had given a scale of the workload of the tasks for the participants in their NASA-TLX scores. After adjusting their sitting position, participants executed their own final experiment, including five tasks and completing NASA-TLX after each task (Fig. 14). They also had a relax time of approximately 3 min before each task. In order to prevent the conflict of the input operation, they were asked to use a mouse and keyboard to complete SYSMON/RESMAN and COMMUN tasks, respectively. Participants' behaviors were recorded through the software itself, a video recorder, and the SMI iView X RED eye tracking system.

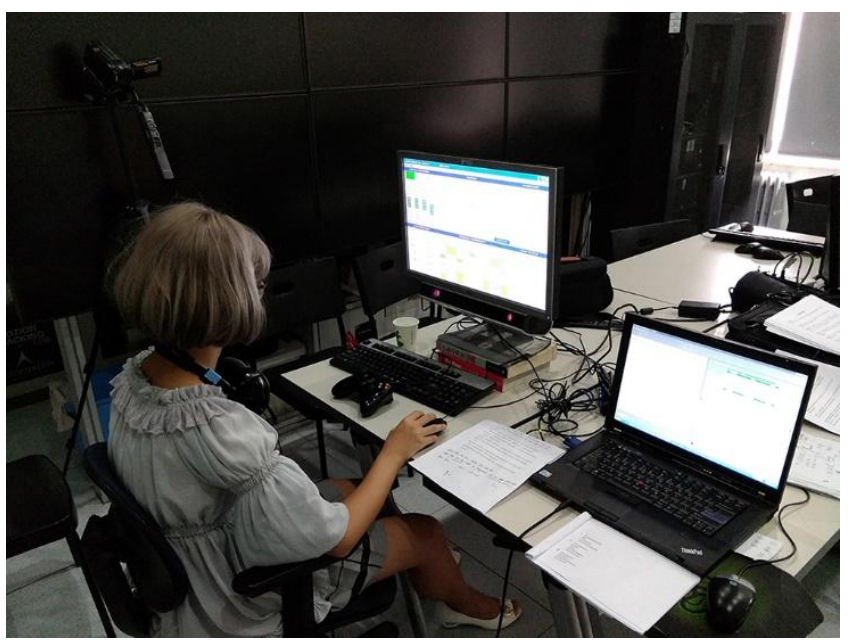

Fig. 14. A participant is doing the experiment

\section{Results}

Table 3 has the interaction behaviors in experiments that were determined by observation, video/eye movement data analysis and the discussion with experts. The method in section 2.1.3 was used to calculate the amount of the V/A-C-P units and to build the CPN model.

\section{Table 3}

The interaction behaviors appeared in experiments.

\begin{tabular}{cc}
\hline Tasks & Interactions \\
\hline SYSMON & F5/F6: V1-C1-P2 \\
TRK & F1/F2/F3/F4: V3-C1-P2 \\
COMMON & V5-C1-P2 \\
RESMON & A4-C1-P5 \\
\hline
\end{tabular}

The ANOVA test results in Table 4 indicate that under different task difficulty and number of subtasks in combination that both NASA-TLX scores and CPN model values were different from each other. Participants experienced higher workloads when task difficulty increased, and also experienced higher workloads when the number of subtasks in combination increased.

Table 4

Results of CPN model values and NASA-TLX scores under different task difficulty and number of subtasks in combinations. Effect sizes are reported as $\eta^{2}$.

\begin{tabular}{cccccccccccc}
\hline Results & & DL1 & DL2 & DL3 & F-value & $\eta^{2}$ & 1TC & 2TCs & 3TCs & F-value & $\eta^{2}$ \\
\hline NASA-TLX & Mean & 33.704 & 36.114 & 47.325 & \multirow{2}{*}{$6.13 * *$} & 0.21 & 18.855 & 40.184 & 56.072 & $75.81^{* * *}$ & 0.73 \\
scores & SD & 10.583 & 16.61 & 15.733 & & & 11.53 & 14.33 & 13.8 & & \\
CPN model & Mean & 189.526 & 285.649 & 409.907 & \multirow{2}{*}{$26.03 * * *$} & 0.43 & 136.438 & 286.218 & 462.114 & $102.32^{* * *}$ & 0.85 \\
$\quad$ values & SD & 103.376 & 143.387 & 125.666 & 26.07 & 112.66 & 107.99 & 10.07 \\
\hline
\end{tabular}


Note: $N=140 ;{ }^{+} p<.1,{ }^{*} p<.05,{ }^{* *} p<.01,{ }^{* * *} p<.001$

DL1 = Difficulty Level 1, DL2= Difficulty Level 2, DL3= Difficulty Level 3, 1TC = 1 Task Combination, 2TC=2 Task Combination, 3TC $=3$ Task Combinations.

1

2

Table 5 shows the mean and SD of NASA-TLX scores and CPN model values of the 14 tasks, respectively. A high correlation coefficient can be observed between the mean NASA-TLX scores and CPN model values, $r=0.9513, p<0.01$, $r_{\mathrm{pb}}=0.948$. The results of multiple regression analysis indicated that CPN model explained $90.5 \%$ of the variance $\left(R^{2}=0.9051, F(1,13)=114.42, p<0.001, f^{2}=0.89\right)$, and its values also significantly predicted NASA-TLX scores $(\beta=0.0988$, $p<0.001)$.

Table 5

Mean and SD of NASA-TLX scores and CPN model values calculated of the 14 tasks.

\begin{tabular}{|c|c|c|c|c|c|c|c|c|}
\hline \multirow[t]{2}{*}{ Tasks } & & \multicolumn{3}{|c|}{ Number of sub-tasks in in combination } & \multicolumn{2}{|c|}{ NASA-TLX Scores } & \multicolumn{2}{|c|}{ CPN Model Values } \\
\hline & & Single & Double & Treble & Mean & SD & Mean & SD \\
\hline \multirow[t]{14}{*}{ Difficulty } & Level1 & COM5 & & & 20.167 & 13.951 & 63.619 & 3.670 \\
\hline & & & COM5\&RES1 & & 32.499 & 11.878 & 192.072 & 17.560 \\
\hline & & & SYS15\&TRK20\% & & 32.283 & 13.087 & 153.031 & 22.978 \\
\hline & & & & SYS15\&RES1\&TRK20\% & 49.868 & 13.620 & 349.388 & 63.597 \\
\hline & Level2 & RES2 & & & 15.4 & 11.191 & 125.600 & 25.788 \\
\hline & & TRK60\% & & & 15.849 & 10.004 & 113.520 & 37.249 \\
\hline & & & RES2\&TRK60\% & & 29.952 & 8.818 & 230.493 & 67.835 \\
\hline & & & COM15\&SYS30 & & 47.468 & 8.512 & 328.569 & 15.214 \\
\hline & & & & RES2\&TRK60\%\&SYS30 & 56.034 & 14.025 & 502.131 & 37.634 \\
\hline & & & & RES2\&TRK60\%\&COM15 & 51.984 & 11.524 & 413.582 & 38.803 \\
\hline & Level3 & SYS45 & & & 24.002 & 7.924 & 243.012 & 16.374 \\
\hline & & & COM25\&TRK100\% & & 43.4 & 9.418 & 355.750 & 41.887 \\
\hline & & & RES3\&SYS45 & & 55.5 & 12.516 & 457.390 & 55.580 \\
\hline & & & & COM25\&RES3\&TRK100\% & 66.4 & 9.225 & 583.475 & 91.019 \\
\hline
\end{tabular}

When executing multiple sub-tasks, participants usually ignored others due to concentrating on one sub-task in a period of time. For the tasks which had two or three sub-tasks in combination and had SYSMON sub-tasks, workload was related to the number of warning icons that participants had ignored. Fig. 15 shows the average ignored number of warning icons of the five tasks that had SYSMON tasks at different NASA-TLX workload ranks of ten participants. It can be observed that in these tasks, participants who experienced lower workloads usually ignored more warning icons.

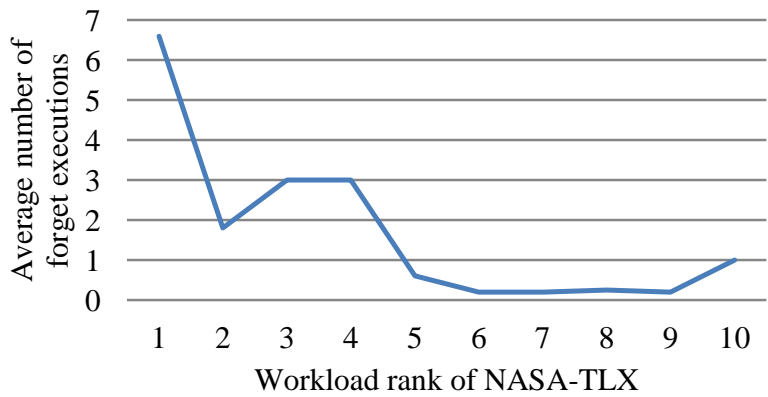

Fig. 15. Average ignored number of warning icons of five tasks that have SYSMON task at different NASA-TLX workload rank

The listening competence of participants was related to their workload in COMMUN tasks. Participants who have attained CET level six $(M=8, S D=2.41 ; M=62.39, S D=11.75$, respectively) experienced lower workloads than those who have attained CET level four both in NASA-TLX scores and CPN model values $(M=32.33, S D=9.35 ; M=86.23, S D=9.10$, respectively) in COM5 task, $t(4)=6.98, p<0.001, r_{p b}=0.961 ; t(4)=5.29, p<0.01, r_{p b}=0.935$, respectively.

The task assignment in this study has a high homogeneity; that is, each task was executed in at least four different age groups by both male and female participants, and in all four time periods of at least five different days. Both the NASA-TLX scores and the CPN model values between males $(M=39.46, S D=13.91 ; M=294.96, S D=147.83$, respectively) and females $(M=38.01, S D=19.85 ; M=290.53, S D=162.62$, respectively) were not significant $(t(13)=0.53, p=n . s$, $r_{p b}=0.145 ; t(13)=0.61, p=n . s, r_{p b}=0.167$ respectively). The implementation time of tasks between AM and PM were not significant $\left(t(13)=-0.21, p=n . s, r_{p b}=0.058 ; t(13)=-0.00048, p=n . s, r_{p b}=0.00013\right.$ respectively). Different educational degrees, that of undergraduate and postgraduate, also had no significant effect on workload $\left(t(13)=1.002, p=n . s, \quad r_{p b}=0.267\right.$; $t(13)=1.409, p=n . s, r_{p b}=0.363$, respectively).

\section{Discussion}

A good accuracy can be observed in the results of the workload evaluation through the CPN model. Accordingly, several issues about the method proposed in this study will be discussed.

\section{Table 6}

Results of workload calculated of task RES2TRK60\%.

\begin{tabular}{lcccccc}
\hline Participants & Ages & Genders & Days & Times & NASA-TLX Scores & CPN model values \\
\hline NO.7 & 25 & F & 3 & 1 & 25 & 185.8
\end{tabular}




\begin{tabular}{|c|c|c|c|c|c|c|c|}
\hline \multirow{9}{*}{$\begin{array}{l}1 \\
2 \\
3 \\
4 \\
5\end{array}$} & NO.3 & 22 & $\mathrm{M}$ & 2 & 1 & 46.67 & 380 \\
\hline & NO.23 & 24 & $\mathrm{M}$ & 7 & 2 & 31.67 & 225.51 \\
\hline & NO.12 & 21 & $\mathrm{M}$ & 8 & 2 & 39 & 287.792 \\
\hline & NO.8 & 20 & M & 6 & 3 & 19.5 & 207.2 \\
\hline & NO.5 & 24 & $\mathrm{~F}$ & 2 & 3 & 20 & 147.2 \\
\hline & NO. 10 & 25 & M & 5 & 4 & 20.67 & 153 \\
\hline & NO.6 & 23 & M & 2 & 4 & 25.17 & 184.2 \\
\hline & NO. 21 & 23 & M & 3 & 4 & 35.67 & 272 \\
\hline & NO. 13 & 21 & $\mathrm{M}$ & 8 & 4 & 36.17 & 262.232 \\
\hline
\end{tabular}

The method proposed in this study could not only evaluate the amount of workload in different task difficulty and sub-tasks combinations but also could distinguish workload in the same task when people varied. An example shown in Table 6 was the results of NASA-TLX scores and the CPN model values of task RES2TRK60\% by its ten participants. In this task, participant 3 had the highest workload, more than twice of the lowest workload of participant 8 , in NASA-TLX scores, and there also existed a notably large difference in their CPN model values. Fig. 16(a) and Fig. 16(b) were the results for the values of sub-task TRK60\% and sub-task RES2 of the two participants. In Fig. 16(a), the black curve represents the values for sub-task TRK60\%, which is the distance between the center of the circle that is constantly moving and the center of the square. From the method proposed in section 2.1.3, the red curve and the green curve represent for $\bar{m}_{T}+\max \left(s_{T}, D_{T \max } / 10\right)$ and $\bar{m}_{T}-\max \left(s_{T}, D_{T \max } / 10\right)$ respectively, where $\bar{m}_{T}$ is average of $N_{\mathrm{T}}$ sequential data inputs of the distance mentioned before, here $N_{\mathrm{T}}$ is equal to five. $s_{\mathrm{T}}$ is the standard deviation and $D_{\mathrm{T} m a x}$ is the maximum difference between the values of distance mentioned in $N_{\mathrm{T}}$ the sequential data. The aim of TRK tasks is to adjust the circle to maintain the shortest distance. That is to say, psychomotor should be calculated according to the number of ranges of the black curve that is beyond the red curve. It is clear that the number of ranges in the upper portion is much more than in the lower in Fig. 16(a). This means that participant 3 took more operations to adjust the circle than participant 8 . The same condition can be observed in Fig. 16(b), participant 3 also did more actions in the RES task than participant 8 . The difference of the ranges calculated here in the RES task implies the data inputs are the values of Tank A; thus, the psychomotor should be calculated according to the number of the ranges of the black curve beyond red curve and black curve below green curve.
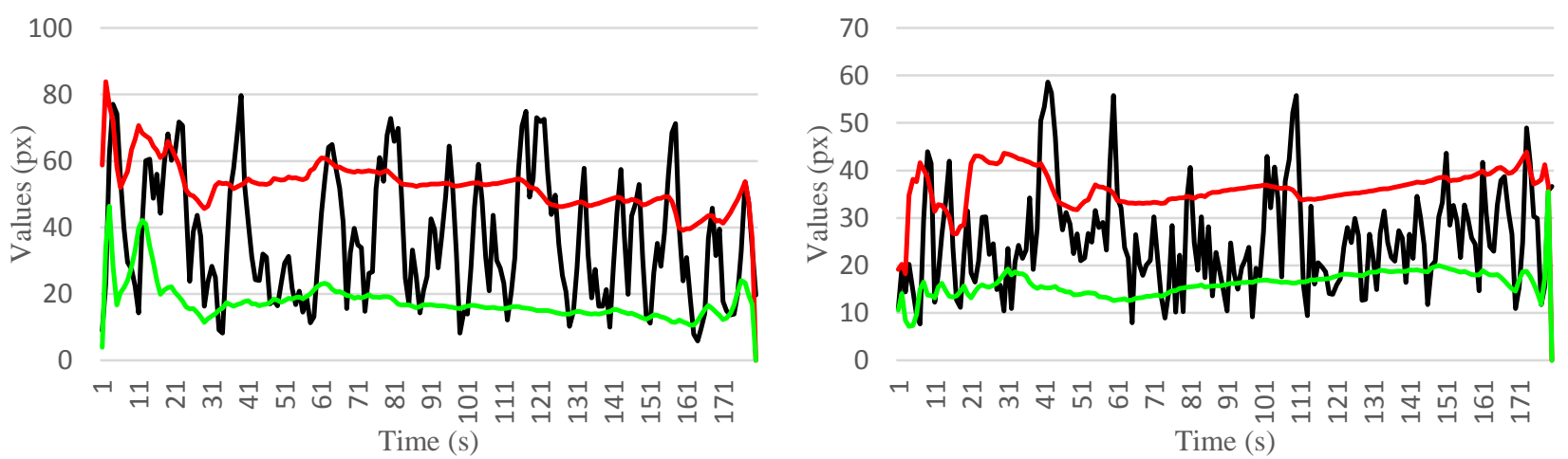

Fig. 16(a). Results of values of sub-task TRK60\% of participant 3(left) and participant 8(right)
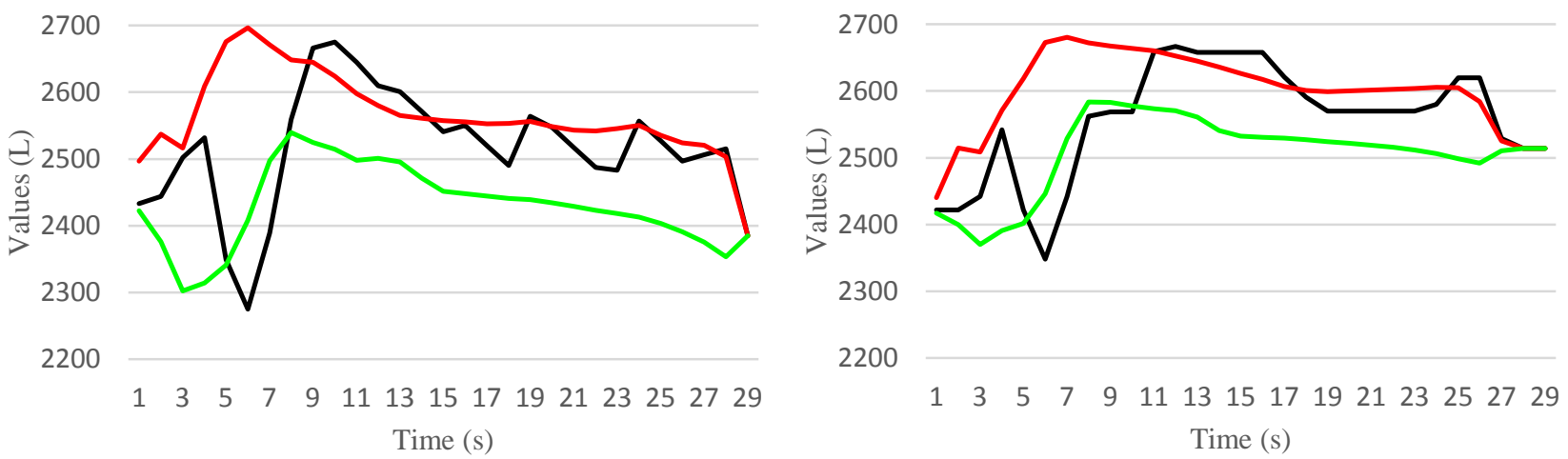

Fig. 16(b). Results of values of Tank A of sub-task RES2 of participant 3(left) and participant 8(right)

Except for the interpretation that participant 3 experienced a higher workload through the analysis of input data, the CPN model can give a more comprehensive description of these two interaction process. Fig. 17(a) and Fig. 17(b) are the CPN models of task RES2TRK60\% of participant 3 and participant 8, respectively. More execution sequences can be observed in the transitions connected to places 'Joystick', 'Button', 'Circle icon' and 'Tank' in Fig. 17(a) than their corresponding transitions in Fig. 17(b). While the transition belonging to place 'Pump' in Fig. 17(b) has two execution sequences more than the corresponding transitions in Fig. 17(a), and it has less contribution to workload because the interaction process of adjusting the pump by perceiving the state of another pump contributes less than the interaction 
process of adjusting the pump by checking up on the value of tanks. Summing up, the CPN model indicates that participant 3 not only did more actions than participant 8 but also some of these actions contributed more to workload.

Next, the method proposed here has given multiple resource theory a new angle, given a more objective usage of

2 3

VACP rating values, and has given strong correlation with NASA-TLX results. Previous studies have used the four dimensions CODES, MODALITIES, STAGES and RESPONSES in multiple resource theory to evaluate workload. In this paper, STAGES is treated as the V/A-C-P units, CODES, MODALITIES and RESPONSES are treated as the components of petri nets, and the four types of petri net structures in Fig. 2 can be observed as the representation of the conflict of each dimension. The usage of VACP rating scales here was, unless the determination of the type of behaviors according to Table1 by experts, the number of behaviors were all determined by objective records. For a better verification of this method here, another form of experiment had been implemented. Two male participants A and B executed all the forty-two tasks in task library. The environment and apparatus were the same as the experiments mentioned before. The difference was that tasks were randomly selected; average was separated to seven parts, and participants completed two parts of twelve tasks in one day. Fig. 18 is the correlation coefficients corresponding of the seven sessions of the two participants. For both participants, the CPN model values were all highly correlated with NASA-TLX scores.

Furthermore, the proposed method can be used not only to evaluate workload but also to reflect the behavior characteristics of different people. Different people will show different interaction behaviors in the same environment, operating equipment and task process. In some areas, such as driving and aircraft piloting, behavioral characteristics can be used as a reference in selecting an operator, establishing guidelines, and designing safety assistant system. Compared with the previous behavior modeling method(Cacciabue et al., 2013; Toledo et al., 2009),the graphical structure of petri nets can give a quantitative and more intuitive representation of the difference between different people. Fig. 19(a) and Fig. 19(b) are part of CPN model of task RES2SYS30COM15 of participant 24 and participant 7 , respectively. Three additional transitions can be observed in Fig. 19(a): one of them is the RR transition belonging to place 'Button', and another two are RT transitions between the places 'Tank' and 'Button'. Through the video recording of the two tasks, we found that when need to adjust the pumps, for participant 24 sometimes moved his gaze from screen to keyboard to find the corresponding buttons, and after pressing the button, his gaze would go back to screen to continue the task. Participant 7 was relatively familiar with the keyboard and he did not exhibit this type of behavior. When different types and amounts of interaction behaviors were implemented by different participants, in CPN model, they were all reflected by different types and amounts of transitions belonging to place, as well as different weights of directed arcs.

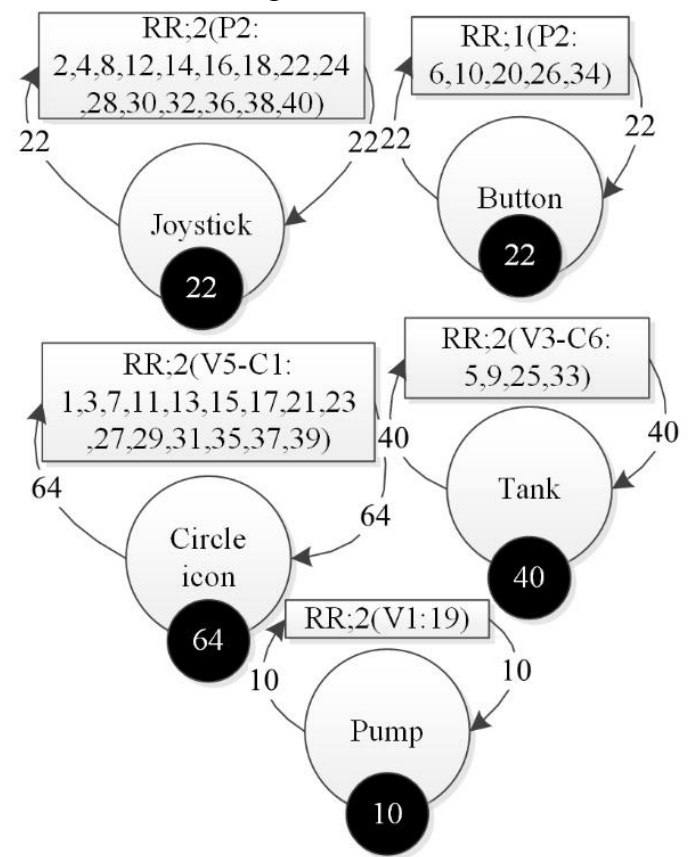

Fig. 17(a). CPN model of task RES2TRK60\% of participant 3 


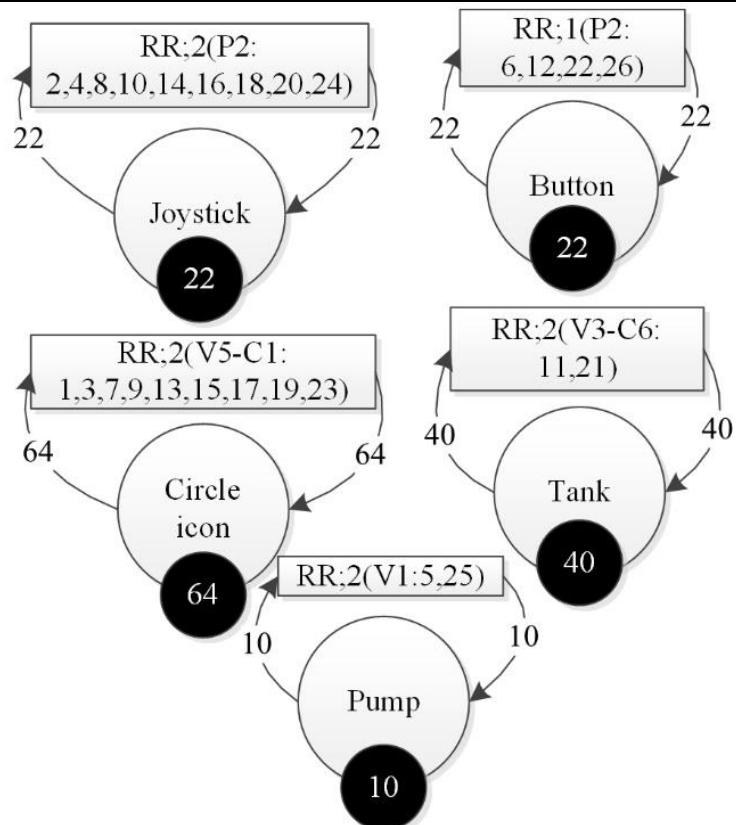

Fig. 17(b). CPN model of task RES2TRK60\% of participant 8

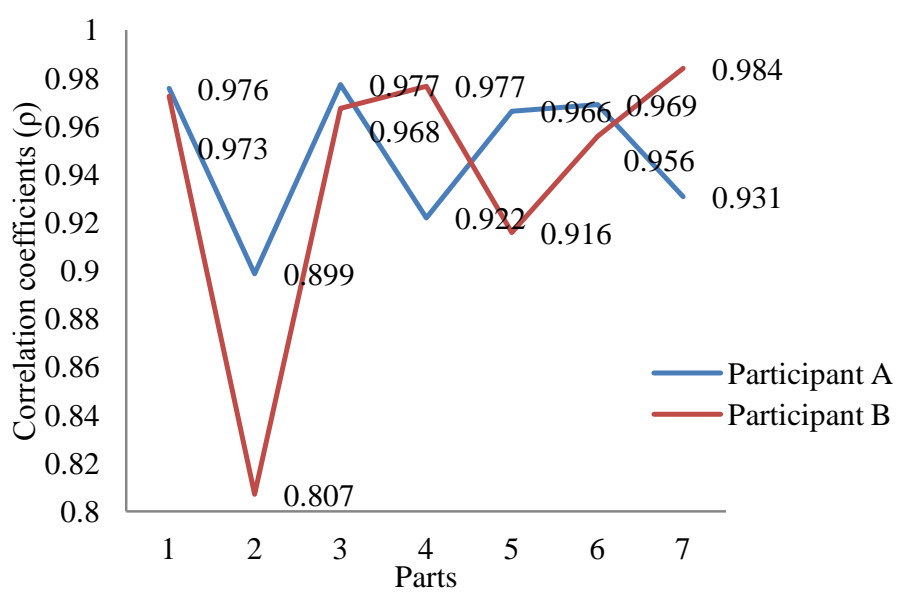

Fig. 18. The correlation coefficients corresponding of the seven sessions of the two participants

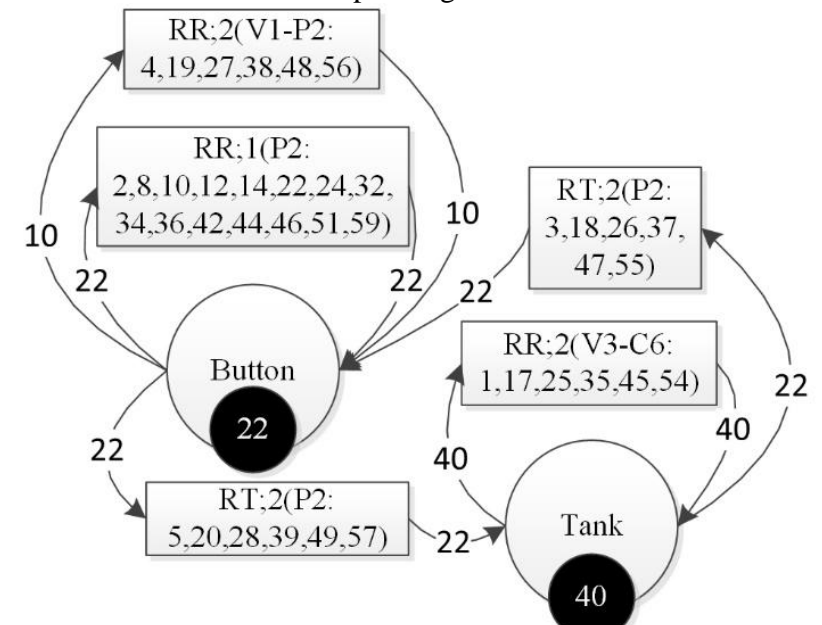

Fig. 19(a). Part of CPN model of task RES2SYS30COM15 of participant 24 


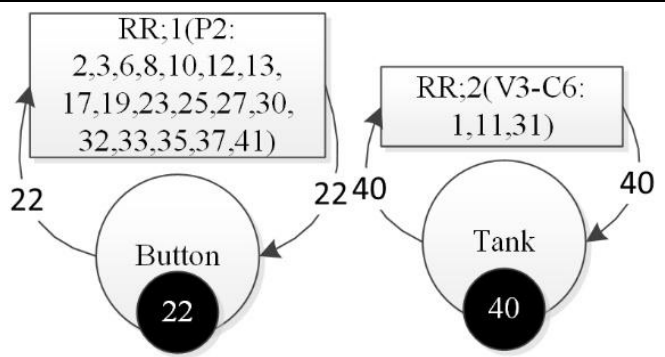

Fig 19(b). Part of CPN model of task RES2SYS30COM15 of participant 7

In addition, except for the supportive results that the large effect size $r_{\mathrm{pb}}$ of the correlation coefficient between mean NASA-TLX scores and CPN model values and $f^{2}$ of the multiple regression analysis, another reason why the CPN model values highly correlated with NASA-TLX scores in both the two experiment mentioned above is that it based on and improved from the multiple resources theory and VACP rating scales which are widely used, and the behavior acquisition methods used in practical application lead to a very low interference on both the participant and task, these make CPN model has a better reliability. A new experiment was executed for a further verification of the repeatability. Four tasks SYS45, COM25TRK100\%, SYS45RES3 and COM25RES3TRK100\% were selected from the fourteen tasks in above experiment, and executed in the same environment by a new group of twenty-seven participants whom with the same requirements but did not take part in the previous experiment mentioned in section 3 . The results indicated that the CPN model values of all the four tasks in experiments before and now did not differ significantly from each other(For SYS45 task, $t(35)=0.381, p=n . s, r_{p b}=0.064$. For COM25TRK100\% task, $t(35)=0.116, p=n . s, r_{p b}=0.02$. For SYS45RES3 task, $t(35)=0.268, p=n . s, r_{p b}=0.045$. For COM25RES3TRK100\% task, $t(35)=0.383, p=n . s, r_{p b}=0.065$.). The sensitivity of CPN model is supported by the large effect size $\eta^{2}$ both in the ANOVA test in Table 4 and the new experiment (For different task combinations $(M=252.796, S D=19.16, M=379.259, S D=33.69, M=579.613, S D=84.69$, respectively, $F(2,80)=245.60$, $\left.p<0.001, \eta^{2}=0.863\right)$, as well as $r_{p b}$ of the T-test of significant difference between CET6 and CET4 in COM5 tasks. This is due to that CPN model is a kind of method based on behavior, more places/transitions/directed arcs will be added in model when new behaviors appear. Self-report measures may conclude the same workload result when participant press button 50 times and 51 times, but the CPN model will have difference. This makes the sensitivity of the model higher.

Lastly, the proposed method has an advantage in pre-evaluation when comparing with the existing subjective self-report measures like NASA-TLX. Only under a carried out experiments with designed tasks, screened participants and prepared experimental tools that can workload be assessed by subjective self-report measures(Carswell et al., 2010; Fallahi et al., 2016). Although the CPN model used in this paper after the completion of experiments, for the tasks which have certain interaction objects and need executed seriously in accordance with the operating rules, like train driving(Dorrian et al., 2011), aircraft controlling(García-Mas et al., 2016) and safety-critical monitoring(Lin et al., 2013), workload could be evaluated through a task analysis by experts. The CPN model can also reflect real-time changes in workload without interrupting task execution for its ability that continuous computing workload values of each moment when new interaction behavior appears. This is also what subjective self-report measures do not possess.

Some future studies should be discussed here. Firstly, through the interview after the experiments, some of the participants expressed that they felt a little bored after completing five tasks. Consequently, increasing time interval or reducing the number of tasks for an individual should be considered in future studies. Secondly, in spite of the high correlation between NASA-TLX and CPN model, the behaviors observed in MATB-II based experiments were just part of the behaviors in VACP rating scales. Therefore, some effective measures should be taken to add more behaviors like speech to ensure a reliable method.

\section{Conclusion}

In this paper, a new method that colored petri nets based on model to evaluate workload was proposed. This method was validated through multitasking environment Multi-Attribute Task Battery-II. The results show that it is highly correlated with NASA-TLX scores. The main results of this study are presented as follows:

(1) A more objective usage of VACP rating scales was given. Except the determination of behavior type, workload was calculated as sum of the number of V/A-C-P units, and the computing method of the amount of V/A-C-P units was also proposed.

(2) A combination of colored petri nets, VACP rating scales and multiple resources theory was put forward. A CPN-based model of interaction from the perspective of resource flow was proposed based on the analysis of CPN structure of multiple resources theory. Seven types V/A-C-P units in CPN model were also involved to different tasks.

(3) The calculation method of workload on the basis of CPN model was proposed. All of the rules and parameters were following the rules of petri nets theory. It has also given a distinction to the effects on different types of transitions and repetitive behaviors. This method can reflect the effects of different behavior style on workload.

(4) The Multi-Attribute Task Battery-II based experiments have proved that the CPN model values of workload were highly correlated with NASA-TLX scores. It can also reflect the behavior characteristics of different people. 


\section{Acknowledgment}

This work is supported by the National Science Foundation of China under Grant 51575037.The authors are very grateful to all the participants who joined in the study.

\section{Reference}

Al-Hakim, L., Maiping, T., Sevdalis, N., 2014. Applying hierarchical task analysis to improving the patient positioning for direct lateral interbody fusion in spinal surgery. Appl. Ergon. 45, 955-966. doi:10.1016/j.apergo.2013.11.013

Benedetto, S., Pedrotti, M., Minin, L., Baccino, T., Re, A., Montanari, R., 2011. Driver workload and eye blink duration. Transp. Res. Part F Traffic Psychol. Behav. 14, 199-208. doi:10.1016/j.trf.2010.12.001

Boles, D.B., Adair, L.P., 2001. The multiple resources questionnaire (MRQ), in: Proceedings of the Human Factors and Ergonomics Society Annual Meeting. SAGE Publications, pp. 1790-1794.

Bromfield, M., Dillman, B., 2015. The Effects of Using an Angle of Attack System on Pilot Performance and Workload during Selected Phases of Flight. Procedia Manuf. 3, 3222-3229. doi:10.1016/j.promfg.2015.07.873

Brookhuis, K.A., de Waard, D., 2010. Monitoring drivers' mental workload in driving simulators using physiological measures. Accid. Anal. Prev. 42, 898-903. doi:10.1016/j.aap.2009.06.001

Brookhuis, K.A., van Driel, C.J.G., Hof, T., van Arem, B., Hoedemaeker, M., 2009. Driving with a congestion assistant; mental workload and acceptance. Appl. Ergon., Psychophysiology in Ergonomics 40, 1019-1025. doi:10.1016/j.apergo.2008.06.010

Cacciabue, P.C., Enjalbert, S., Söderberg, H., Tapani, A., 2013. Unified Driver Model simulation and its application to the automotive, rail and maritime domains. Transp. Res. Part F Traffic Psychol. Behav. 21, 315-327. doi:10.1016/j.trf.2013.09.020

Carswell, C.M., Lio, C.H., Grant, R., Klein, M.I., Clarke, D., Seales, W.B., Strup, S., 2010. Hands-free administration of subjective workload scales: Acceptability in a surgical training environment. Appl. Ergon. 42, 138-145. doi:10.1016/j.apergo.2010.06.003

Chen, S.-W., Fang, C.-Y., Tien, C.-T., 2013. Driving behaviour modelling system based on graph construction. Transp. Res. Part C Emerg. Technol. 26, 314-330. doi:10.1016/j.trc.2012.10.004

Comstock, J.R., Arnegard, R.J., 1992. The multi-attribute task battery for human operator workload and strategic behavior research.

de Winter, J.C.F., Happee, R., Martens, M.H., Stanton, N.A., 2014. Effects of adaptive cruise control and highly automated driving on workload and situation awareness: A review of the empirical evidence. Transp. Res. Part F Traffic Psychol. Behav. 27, 196-217. doi:10.1016/j.trf.2014.06.016

DiDomenico, A., Nussbaum, M.A., 2011. Effects of different physical workload parameters on mental workload and performance. Int. J. Ind. Ergon. 41, 255-260. doi:10.1016/j.ergon.2011.01.008

DiDomenico, A., Nussbaum, M.A., 2005. Interactive effects of mental and postural demands on subjective assessment of mental workload and postural stability. Saf. Sci. 43, 485-495. doi:10.1016/j.ssci.2005.08.010

Di Stasi, L.L., Antolí, A., Gea, M., Cañas, J.J., 2011. A neuroergonomic approach to evaluating mental workload in hypermedia interactions. Int. J. Ind. Ergon. 41, 298-304. doi:10.1016/j.ergon.2011.02.008

Dorrian, J., Baulk, S.D., Dawson, D., 2011. Work hours, workload, sleep and fatigue in Australian Rail Industry employees. Appl. Ergon. 42, 202-209. doi:10.1016/j.apergo.2010.06.009

Fallahi, M., Motamedzade, M., Heidarimoghadam, R., Soltanian, A.R., Miyake, S., 2016. Effects of mental workload on physiological and subjective responses during traffic density monitoring: A field study. Appl. Ergon. 52, 95-103. doi:10.1016/j.apergo.2015.07.009

García-Mas, A., Ortega, E., Ponseti, J., de Teresa, C., Cárdenas, D., 2016. Workload and cortisol levels in helicopter combat pilots during simulated flights. Rev. Andal. Med. Deporte 9, 7-11. doi:10.1016/j.ramd.2015.12.001

Gonzalez, C., 2005. Task workload and cognitive abilities in dynamic decision making. Hum. Factors 47, 92-101. doi:10.1518/0018720053653767

Hamilton, W.I., Clarke, T., 2005. Driver performance modelling and its practical application to railway safety. Appl. Ergon. 36, 661-670. doi:10.1016/j.apergo.2005.07.005

Hart, S.G., Staveland, L.E., 1988. Development of NASA-TLX (Task Load Index): Results of Empirical and Theoretical Research, in: Meshkati, P.A.H. and N. (Ed.), Advances in Psychology, Human Mental Workload. North-Holland, pp. 139-183.

Jo, S., Myung, R., Yoon, D., 2012. Quantitative prediction of mental workload with the ACT-R cognitive architecture. Int. J. Ind. Ergon. 42, 359-370. doi:10.1016/j.ergon.2012.03.004

Jung, H.S., Jung, H.-S., 2001. Establishment of overall workload assessment technique for various tasks and workplaces. Int. J. Ind. Ergon. 28, 341-353. doi:10.1016/S0169-8141(01)00040-3

Keller, J., 2002. Human performance modeling for discrete-event simulation: workload, in: Simulation Conference, 2002. Proceedings of the Winter. Presented at the Simulation Conference, 2002. Proceedings of the Winter, pp. 157-162 vol.1. doi:10.1109/WSC.2002.1172879 
Kontogiannis, T., 2005. Integration of task networks and cognitive user models using coloured Petri nets and its application to job design for safety and productivity. Cogn. Technol. Work 7, 241-261. doi:10.1007/s10111-005-0010-z

Kontogiannis, T., 2003. A Petri Net-based approach for ergonomic task analysis and modeling with emphasis on adaptation to system changes. Saf. Sci. 41, 803-835. doi:10.1016/S0925-7535(02)00035-8

Kontogiannis, T., Leopoulos, V., Marmaras, N., 2000. A comparison of accident analysis techniques for safety-critical man-machine systems. Int. J. Ind. Ergon. 25, 327-347.

Lei, S., Welke, S., Roetting, M., 2009. Representation of driver's mental workload in EEG data. Hum. Factors Secur. Saf. 285-294.

Liang, S.-F.M., Rau, C.-L., Tsai, P.-F., Chen, W.-S., 2014. Validation of a task demand measure for predicting mental workloads of physical therapists. Int. J. Ind. Ergon. 44, 747-752. doi:10.1016/j.ergon.2014.08.002

Lin, C.J., Yenn, T.-C., Jou, Y.-T., Hsieh, T.-L., Yang, C.-W., 2013. Analyzing the staffing and workload in the main control room of the advanced nuclear power plant from the human information processing perspective. Saf. Sci. 57, 161-168. doi:10.1016/j.ssci.2013.02.004

Mascaro, S., Asada, H.H., 1998. Hand-in-glove human-machine interface and interactive control: task process modeling using dual Petri nets, in: 1998 IEEE International Conference on Robotics and Automation, 1998. Proceedings. Presented at the 1998 IEEE International Conference on Robotics and Automation, 1998. Proceedings, pp. 1289-1295 vol.2. doi:10.1109/ROBOT.1998.677281

McCracken, J.H., Aldrich, T.B., ANACAPA SCIENCES INC FORT RUCKER AL, 1984. Analyses of Selected LHX Mission Functions: Implications for Operator Workload and System Automation Goals. Defense Technical Information Center, Ft. Belvoir.

Mcllroy, R.C., Stanton, N.A., 2015. A decision ladder analysis of eco-driving: the first step towards fuel-efficient driving behaviour. Ergonomics 58, 866-882. doi:10.1080/00140139.2014.997807

Moray, N., 2013. Mental Workload: Its Theory and Measurement. Springer Science \& Business Media.

North, R.A., Riley, V.A., 1989. W/INDEX: A Predictive Model of Operator Workload, in: McMillan, G.R., Beevis, D., Salas, E., Strub, M.H., Sutton, R., Breda, L.V. (Eds.), Applications of Human Performance Models to System Design. Springer US, pp. 81-89.

Overmeyer, L., Podszus, F., Dohrmann, L., 2016. Multimodal speech and gesture control of AGVs, including EEG-based measurements of cognitive workload. CIRP Ann. - Manuf. Technol. 65, 425-428. doi:10.1016/j.cirp.2016.04.030

Oyewole, S.A., Haight, J.M., 2011. Determination of optimal paths to task goals using expert system based on GOMS model. Comput. Hum. Behav. 27, 823-833. doi:10.1016/j.chb.2010.11.007

Pauzié, A., Manzano, J., 2007. Evaluation of driver mental workload facing new in-vehicle information and communication technology, in: Proceedings of the 20th Enhanced Safety of Vehicles Conference (ESV20), Lyon, France.

Pickup, L., Wilson, J., Lowe, E., 2010. The Operational Demand Evaluation Checklist (ODEC) of workload for railway signalling. Appl. Ergon. 41, 393-402. doi:10.1016/j.apergo.2009.09.003

Pickup, L., Wilson, J.R., Norris, B.J., Mitchell, L., Morrisroe, G., 2005. The Integrated Workload Scale (IWS): A new self-report tool to assess railway signaller workload. Appl. Ergon. 36, 681-693. doi:10.1016/j.apergo.2005.05.004

Popkin, S., Gertler, J., Reinach, S., 2001. A PRELIMINARY EXAMINATION OF RAILROAD DISPATCHER WORKLOAD, STRESS, AND FATIGUE.

Reid, G.B., Nygren, T.E., 1988. The Subjective Workload Assessment Technique: A Scaling Procedure for Measuring Mental Workload, in: Meshkati, P.A.H. and N. (Ed.), Advances in Psychology, Human Mental Workload. North-Holland, pp. 185-218.

Rubio, S., Díaz, E., Martín, J., Puente, J.M., 2004. Evaluation of Subjective Mental Workload: A Comparison of SWAT, NASA-TLX, and Workload Profile Methods. Appl. Psychol. 53, 61-86. doi:10.1111/j.1464-0597.2004.00161.x

Santiago-Espada, Y., Myer, R.R., Latorella, K.A., Comstock, J.R., 2011. The Multi-Attribute Task Battery II (MATB-II) Software for Human Performance and Workload Research: A User's Guide.

Shneiderman, B., 2012. Handbook of Human Factors and Ergonomics (4th ed.). Int. J. Human-Computer Interact. 28, 838-838. doi:10.1080/10447318.2012.701542

Silva, F.P. da, 2014. Mental Workload, Task Demand and Driving Performance: What Relation? Procedia - Soc. Behav. Sci. 162, 310-319. doi:10.1016/j.sbspro.2014.12.212

Toledo, T., Koutsopoulos, H.N., Ben-Akiva, M., 2009. Estimation of an integrated driving behavior model. Transp. Res. Part C Emerg. Technol. 17, 365-380. doi:10.1016/j.trc.2009.01.005

Wang, J., Fang, W., 2014. A structured method for the traffic dispatcher error behavior analysis in metro accident investigation. Saf. Sci. 70, 339-347. doi:10.1016/j.ssci.2014.07.014

Wang, Z., Hope, R.M., Wang, Z., Ji, Q., Gray, W.D., 2012. Cross-subject workload classification with a hierarchical Bayes model. NeuroImage 59, 64-69. doi:10.1016/j.neuroimage.2011.07.094

Wen, C.-H., Hwang, S.-L., 1999. A graphic modeling and analysis tool for human fault diagnosis tasks. Int. J. Ind. Ergon. 23, 67-81. doi:10.1016/S0169-8141(97)00102-9

Wickens, C.D., 2002. Multiple resources and performance prediction. Theor. Issues Ergon. Sci. 3, 159-177. doi:10.1080/14639220210123806 
Yagoda, R.E., Coovert, M.D., 2012. How to work and play with robots: An approach to modeling human-robot interaction. Comput. Hum. Behav. 28, 60-68. doi:10.1016/j.chb.2011.08.011

1

2

3

4

5

6

7

8

9

10

11

12

13

14

15

16

17

18

19

20

21

22

23

24

25

26

27

28

29

30

31

32

33

34

35

36

37

38

39

40

41

42

43

44

45

46

47

48

49

50

51

52

53

54

55

56

57

58

59

60

61

62

63

64

65

Zarjam, P., Epps, J., Chen, F., Lovell, N.H., 2013. Estimating cognitive workload using wavelet entropy-based features during an arithmetic task. Comput. Biol. Med. 43, 2186-2195. doi:10.1016/j.compbiomed.2013.08.021 